\title{
Research Article \\ Existence Theorems for First-Order Equations on Time Scales with $\Delta$-Carathéodory Functions
}

\author{
Hugues Gilbert \\ Département de Mathématiques, Collège Édouard-Montpetit, 945 Chemin de Chambly, Longueuil, \\ QC, Canada J4H 3M6
}

Correspondence should be addressed to Hugues Gilbert, hugues.gilbert@college-em.qc.ca

Received 14 June 2010; Accepted 3 December 2010

Academic Editor: Kanishka Perera

Copyright (C) 2010 Hugues Gilbert. This is an open access article distributed under the Creative Commons Attribution License, which permits unrestricted use, distribution, and reproduction in any medium, provided the original work is properly cited.

This paper concerns the existence of solutions for two kinds of systems of first-order equations on time scales. Existence results for these problems are obtained with new notions of solution tube adapted to these systems. We consider the general case where the right member of the system is $\Delta$-Carathéodory and, hence, not necessarily continuous.

\section{Introduction}

In this paper, we establish existence results for the following systems:

$$
\begin{aligned}
& x^{\Delta}(t)=f(t, x(\sigma(t))), \quad \Delta \text {-a.e. } t \in \mathbb{T}_{0}, x \in(B C) ; \\
& x^{\Delta}(t)=f(t, x(t)), \quad \Delta \text {-a.e. } t \in \mathbb{T}_{0}, x(a)=x(b) .
\end{aligned}
$$

Here, $\mathbb{T}$ is an arbitrary compact time scale where we note $a=\min \mathbb{T}, b=\max \mathbb{T}$, and $\mathbb{T}_{0}=$ $\mathbb{T} \backslash\{b\}$. Moreover, $f: \mathbb{T}_{0} \times \mathbb{R}^{n} \rightarrow \mathbb{R}^{n}$ is a $\Delta$-Carathéodory function and $(B C)$ denotes one of the following boundary conditions:

$$
\begin{gathered}
x(a)=x_{0}, \\
x(a)=x(b) .
\end{gathered}
$$

In the literature, this kind of problem was mainly treated for $n=1$. The existence of extremal solutions was established in $[1,2]$. Moreover, some existence results in the particular 
case where the time scale is a discrete set (difference equation) were obtained with the lower and upper solution method as in $[3,4]$. In this paper, we introduce a new notion which generalizes to systems of first-order equations on time scales the notions of lower and upper solutions. This notion called solution tube for system (1.1) (resp., (1.2)) will be useful to get a new existence result for (1.1) (resp., (1.2)). Our notion of solution tube is in the spirit of the notion of solution tube for systems of first-order differential equations introduced in [5]. Our notion is new even in the case of systems of first-order difference equations. In this case, we generalize to the systems a result of [3] for equation (1.2).

Some papers treat the existence of solutions to systems of first-order equations on time scales. Existence results are obtained in $[6,7]$ under hypothesis different from ours. However, some particular cases obtained in [7] are corollaries of our existence result for problem (1.1). Also, our existence results treat the case where the right members in (1.1) and (1.2) are $\Delta$ Carathéodory functions which are more general than continuous functions used for systems studied in [6,7]. Let us mention that existence of extremal solutions for infinite systems of first-order equations of time scale with $\Delta$-Carathéodory functions is established in [8].

This paper is organized as follows. The third section presents an existence result for the problem (1.1), and in the last section, we obtain an existence theorem for the problem (1.2). We start with some notations, definitions, and results on time scales equations which are used throughout this paper.

\section{Preliminaries and Notations}

In this section, we establish notations, definitions, and results on equations on time scales which are used throughout this paper. The reader may consult [9-11] and the references therein to find the proofs and to get a complete introduction to this subject.

Let $\mathbb{T}$ be a time scale, which is a closed nonempty subset of $\mathbb{R}$. For $t \in \mathbb{T}$, we define the forward jump operator $\sigma: \mathbb{T} \rightarrow \mathbb{T}$ (resp., the backward jump operator $\rho: \mathbb{T} \rightarrow \mathbb{T}$ ) by $\sigma(t)=$ $\inf \{s \in \mathbb{T}: s>t\}$ (resp., by $\rho(t)=\sup \{s \in \mathbb{T}: s<t\}$ ). We suppose that $\sigma(t)=t$ if $t$ is the maximum of $\mathbb{T}$ and that $\rho(t)=t$ if $t$ is the minimum of $\mathbb{T}$. We say that $t$ is right-scattered (resp., left-scattered) if $\sigma(t)>t$ (resp., if $\rho(t)<t$ ). We say that $t$ is isolated if it is right-scattered and left-scattered. Also, if $t<\sup \mathbb{T}$ and $\sigma(t)=t$, we say that $t$ is right-dense. If $t>\inf \mathbb{T}$ and $\rho(t)=t$, we say that $t$ is left dense. Points that are right dense and left dense are called dense. The graininess function $\mu: \mathbb{T} \rightarrow[0, \infty)$ is defined by $\mu(t)=\sigma(t)-t$.

If $\mathbb{T}$ has a left-scattered maximum, then $\mathbb{T}^{\mathcal{K}}=\mathbb{T} \backslash\{\sup \mathbb{T}\}$. Otherwise, $\mathbb{T}^{\mathcal{K}}=\mathbb{T}$. In summary,

$$
\mathbb{T}^{\kappa}= \begin{cases}\mathbb{T} \backslash(\rho(\sup \mathbb{T}), \sup \mathbb{T}] & \text { if } \sup \mathbb{T}<\infty \\ \mathbb{T} & \text { if } \sup \mathbb{T}=\infty\end{cases}
$$

If $\mathbb{T}$ is bounded, then $\mathbb{T}_{0} \subset \mathbb{T}^{\kappa}$ where $\mathbb{T}_{0}:=\mathbb{T} \backslash\{\max \mathbb{T}\}$.

Definition 2.1. Assume $f: \mathbb{T} \rightarrow \mathbb{R}^{n}$ is a function and let $t \in \mathbb{T}^{\kappa}$. We say that $f$ is $\Delta$-differentiable at $t$ if there exists a vector $f^{\Delta}(t) \in \mathbb{R}^{n}$ such that for all $\epsilon>0$, there exists a neighborhood $U$ of $t$, where

$$
\left\|\left(f(\sigma(t))-f(s)-f^{\Delta}(t)(\sigma(t)-s)\right)\right\| \leq \epsilon|\sigma(t)-s|
$$


for every $s \in U$. We call $f^{\Delta}(t)$ the $\Delta$-derivative of $f$ at $t$. If $f$ is $\Delta$-differentiable at $t$ for every $t \in \mathbb{T}^{\kappa}$, then $f^{\Delta}: \mathbb{T}^{\kappa} \rightarrow \mathbb{R}^{n}$ is called the $\Delta$-derivative of $f$ on $\mathbb{T}^{\kappa}$.

Theorem 2.2. Assume $f: \mathbb{T} \rightarrow \mathbb{R}^{n}$ is a function and let $t \in \mathbb{T}^{\kappa}$. Then, we have the following.

(i) If $f$ is $\Delta$-differentiable at then $f$ is continuous at $t$.

(ii) If $f$ is continuous at $t$ and if $t$ is right-scattered, then $f$ is $\Delta$-differentiable at $t$ and

$$
f^{\Delta}(t)=\frac{f(\sigma(t))-f(t)}{\mu(t)}
$$

(iii) If $t$ is right dense, then $f$ is $\Delta$-differentiable at $t$ if and only if $\lim _{s \rightarrow t}((f(t)-f(s)) /(t-s))$ exists in $\mathbb{R}^{n}$. In this case, $f^{\Delta}(t)=\lim _{s \rightarrow t}((f(t)-f(s)) /(t-s))$.

(iv) If $f$ is $\Delta$-differentiable at $t$, then $f(\sigma(t))=f(t)+\mu(t) f^{\Delta}(t)$.

Theorem 2.3. If $f, g: \mathbb{T} \rightarrow \mathbb{R}$ are $\Delta$-differentiable at $t \in \mathbb{T}^{\kappa}$, then

(i) $f+g$ is $\Delta$-differentiable at $t$ and $(f+g)^{\Delta}(t)=f^{\Delta}(t)+g^{\Delta}(t)$,

(ii) $\alpha f$ is $\Delta$-differentiable at $t$ for every $\alpha \in \mathbb{R}$ and $(\alpha f)^{\Delta}(t)=\alpha f^{\Delta}(t)$,

(iii) $f g$ is $\Delta$-differentiable at $t$ and $(f g)^{\Delta}(t)=f^{\Delta}(t) g(t)+f(\sigma(t)) g^{\Delta}(t)=f(t) g^{\Delta}(t)+$ $f^{\Delta}(t) g(\sigma(t))$,

(iv) If $g(t) g(\sigma(t)) \neq 0$, then $f / g$ is $\Delta$-differentiable at $t$ and

$$
\left(\frac{f}{g}\right)^{\Delta}(t)=\frac{f^{\Delta}(t) g(t)-f(t) g^{\Delta}(t)}{g(t) g(\sigma(t))}
$$

The next result is an adaptation of Theorem 1.87 in [10].

Theorem 2.4. Let $W$ be an open set of $\mathbb{R}^{n}$ and $t \in \mathbb{T}$ a right-dense point. If $g: \mathbb{T} \rightarrow \mathbb{R}^{n}$ is $\Delta$ differentiable at $t$ and if $f: W \rightarrow \mathbb{R}$ is differentiable at $g(t) \in W$, then $f \circ g$ is $\Delta$-differentiable at $t$ and $(f \circ g)^{\Delta}(t)=\left\langle f^{\prime}(g(t)), g^{\Delta}(t)\right\rangle$.

Example 2.5. Assume $x: \mathbb{T} \rightarrow \mathbb{R}^{n}$ is $\Delta$-differentiable at $t \in \mathbb{T}$. We know that $\|\cdot\|$ : $\mathbb{R}^{n} \backslash\{0\} \rightarrow[0, \infty)$ is differentiable. If $t=\sigma(t)$, by the previous theorem, we have $\|x(t)\|^{\Delta}=$ $\left\langle x(t), x^{\Delta}(t)\right\rangle /\|x(t)\|$.

Definition 2.6. A function $f: \mathbb{T} \rightarrow \mathbb{R}^{n}$ is called rd-continuous provided it is continuous at right-dense points in $\mathbb{T}$ and its left-sided limits exist (finite) at left-dense points in $\mathbb{T}$. The set of rd-continuous functions $f: \mathbb{T} \rightarrow \mathbb{R}^{n}$ is denoted by $C_{\mathrm{rd}}\left(\mathbb{T}, \mathbb{R}^{n}\right)$. The set of functions $f: \mathbb{T} \rightarrow \mathbb{R}^{n}$ that are $\Delta$-differentiable and whose $\Delta$-derivative is rd-continuous is denoted by $C_{\mathrm{rd}}^{1}\left(\mathbb{T}, \mathbb{R}^{n}\right)$.

It is possible to define a theory of measure and integration for an arbitrary bounded time scale $\mathbb{T}$ where $a=\min \mathbb{T}<\max \mathbb{T}=b$. We recall the notion of $\Delta$-measure as introduced in chapter 5 of [9]. Define $\mathcal{F}_{1}$ the family of intervals of $\mathbb{T}$ of the form

$$
[c, d)=\{t \in \mathbb{T}: c \leq t<d\},
$$


where $c, d \in \mathbb{T}$ and $c \leq d$. The interval $[c, c)$ is understood as the empty set. An outer measure $m_{1}^{*}$ on $D(\mathbb{T})$ is defined as follows: for $E \subset \mathbb{T}$,

$$
m_{1}^{*}(E)= \begin{cases}\inf \left\{\sum_{k=1}^{m}\left(d_{k}-c_{k}\right): E \subset \bigcup_{k=1}^{m}\left[c_{k}, d_{k}\right) \text { with }\left[c_{k}, d_{k}\right) \in \mathcal{F}_{1}\right\} & \text { if } b \notin E \\ \infty & \text { if } b \in E\end{cases}
$$

Definition 2.7. A set $A \subset \mathbb{T}$ is said to be $\Delta$-measurable if for every set $E \subset \mathbb{T}$,

$$
m_{1}^{*}(E)=m_{1}^{*}(E \cap A)+m_{1}^{*}(E \cap(\mathbb{T} \backslash A)) .
$$

Now, denote

$$
\mathcal{M}\left(m_{1}^{*}\right):=\{A \subset \mathbb{T}: A \text { is } \Delta \text {-measurable }\}
$$

The Lebesgue $\Delta$-measure on $\mathcal{M}\left(m_{1}^{*}\right)$, denoted by $\mu_{\Delta}$, is the restriction of $m_{1}^{*}$ to $\mathcal{M}\left(m_{1}^{*}\right)$. We get a complete measurable space with $\left(\mathbb{T}, \mathcal{M}\left(m_{1}^{*}\right), \mu_{\Delta}\right)$.

With this definition of complete measurable space for a bounded time scale $\mathbb{T}$, we can define the notions of $\Delta$-measurability and $\Delta$-integrability for functions $f: \mathbb{T} \rightarrow \mathbb{R}$ following the same ideas of the theory of Lebesgue integral. We omit here these definitions that an interested reader can find in [12]. We only present definitions and results which will be useful for this paper.

Definition 2.8. Let $E \subset \mathbb{T}$ be a $\Delta$-measurable set and $f: \mathbb{T} \rightarrow \mathbb{R}$ be a $\Delta$-measurable function. We say that $f \in L_{\Delta}^{1}(E)$ provided

$$
\int_{E}|f(s)| \Delta s<\infty
$$

We say that a $\Delta$-measurable function $f: \mathbb{T} \rightarrow \mathbb{R}^{n}$ is in the set $L_{\Delta}^{1}\left(E, \mathbb{R}^{n}\right)$ provided

$$
\int_{E}\left|f_{i}(s)\right| \Delta s<\infty
$$

for each of its components $f_{i}: \mathbb{T} \rightarrow \mathbb{R}$.

Proposition 2.9. Assume $f \in L_{\Delta}^{1}\left(E, \mathbb{R}^{n}\right)$. Then,

$$
\left\|\int_{E} f(s) \Delta s\right\| \leq \int_{E}\|f(s)\| \Delta s
$$

Many results of integration theory are established for measurable functions $f: X \rightarrow \mathbb{R}$ where $(X, \tau, \mu)$ is a complete measurable space. These results are in particular true for the measurable space $\left(\mathbb{T}, \mathcal{M}\left(m_{1}^{*}\right), \mu_{\Delta}\right)$. We recall two results of the theory of integration adapted to our situation. 
Theorem 2.10 (Lebesgue-dominated convergence Theorem). Let $\left\{f_{n}\right\}_{n \in \mathbb{N}}$ be a sequence of functions in $L_{\Delta}^{1}\left(\mathbb{T}_{0}\right)$. If there exists a function $f: \mathbb{T}_{0} \rightarrow \mathbb{R}$ such that $f_{n}(t) \rightarrow f(t) \Delta$-a.e. $t \in \mathbb{T}_{0}$ and if there exists a function $g \in L_{\Delta}^{1}\left(\mathbb{T}_{0}\right)$ such that $\left\|f_{n}(t)\right\| \leq g(t) \Delta$-a.e. $t \in \mathbb{T}_{0}$ and for every $n \in \mathbb{N}$, then $f_{n} \rightarrow f$ in $L_{\Delta}^{1}\left(\mathbb{T}_{0}\right)$.

Theorem 2.11. The set $L_{\Delta}^{1}\left(\mathbb{T}_{0}\right)$ is a Banach space endowed with the norm $\|f\|_{L_{\Delta}^{1}}:=\int_{\mathbb{T}_{0}}|f(s)| \Delta s$.

The following results were obtained in [12] where a useful relation between the $\Delta$ measure on $\mathbb{T}$ (resp., $\Delta$-integral on $\mathbb{T}$ ) and the Lebesgue measure $\left(\mu_{L}\right)$ on $\mathbb{R}$ (resp., Lebesgue integral on $\mathbb{R}$ ) is established. To establish these results, the authors of [12] prove that the set of right-scattered points of $\mathbb{T}$ is at most countable. Then, there are a set of index $I \subset \mathbb{N}$ and a set $\left\{t_{i}\right\}_{i \in I} \subset \mathbb{T}$ such that $R_{\mathbb{T}}:=\{t \in \mathbb{T}: t<\sigma(t)\}=\left\{t_{i}\right\}_{i \in I}$.

Proposition 2.12. Let $A \subset \mathbb{T}$. Then, $A$ is $\Delta$-measurable if and only if $A$ is Lebesgue measurable. In such a case, the following properties hold for every $\Delta$-measurable set $A$.

(i) If $b \notin A$, then

$$
\mu_{\Delta}(A)=\Sigma_{i \in I_{A}}\left(\sigma\left(t_{i}\right)-t_{i}\right)+\mu_{L}(A)
$$

(ii) $\mu_{\Delta}(A)=\mu_{L}(A)$ if and only if $b \notin A$ and $A$ has no right-scattered points. Here, $I_{A}=\{i \in$ $\left.I: t_{i} \in R_{\mathbb{T}} \cap A\right\}$.

To establish the relation between $\Delta$-integration on $\mathbb{T}$ and Lebesgue integration on a real compact interval, the function $f: \mathbb{T} \rightarrow \mathbb{R}$ is extended to $[a, b]$ in the following way.

$$
\widehat{f}(t):= \begin{cases}f(t), & \text { if } t \in \mathbb{T}, \\ f\left(t_{i}\right), & \text { if } t \in\left(t_{i}, \sigma\left(t_{i}\right)\right), \text { for an } i \in I_{\mathbb{T}}\end{cases}
$$

Theorem 2.13. Let $E \subset \mathbb{T}$ be a $\Delta$-measurable set such that $b \notin E$ and let $\widehat{E}=E \cup \bigcup_{i \in I_{E}}\left(t_{i}, \sigma\left(t_{i}\right)\right)$. Let $f: \mathbb{T} \rightarrow \mathbb{R}$ be a $\Delta$-measurable function and $\widehat{f}:[a, b] \rightarrow \mathbb{R}$ its extension on $[a, b]$. Then, $f$ is $\Delta$-integrable on $E$ if and only if $\widehat{f}$ is Lebesgue integrable on $\widehat{E}$. In such a case, one has

$$
\int_{E} f(s) \Delta s=\int_{\widehat{E}} \widehat{f}(s) d s
$$

Also, the function $f: \mathbb{T} \rightarrow \mathbb{R}$ can be extended on $[a, b]$ in another way. Define $\bar{f}(t):$ $[a, b] \rightarrow \mathbb{R}$ by

$$
\bar{f}(t):= \begin{cases}f(t), & \text { if } t \in \mathbb{T}, \\ f\left(t_{i}\right)+\frac{f\left(\sigma\left(t_{i}\right)\right)-f\left(t_{i}\right)}{\mu\left(t_{i}\right)}\left(t-t_{i}\right), & \text { if } t \in\left(t_{i}, \sigma\left(t_{i}\right)\right), \text { for an } i \in I_{\mathbb{T}} .\end{cases}
$$

Definition 2.14. A function $f: \mathbb{T} \rightarrow \mathbb{R}$ is said to be absolutely continuous on $\mathbb{T}$ if for every $\epsilon>0$, there exists a $\delta>0$ such that if $\left\{\left[a_{k}, b_{k}\right)\right\}_{k=1}^{n}$ with $a_{k}, b_{k} \in \mathbb{T}$ is a finite pairwise disjoint family of subintervals of $\mathbb{T}$ satisfying $\sum_{k=1}^{n}\left(b_{k}-a_{k}\right)<\delta$, then $\Sigma_{k=1}^{n}\left|f\left(b_{k}\right)-f\left(a_{k}\right)\right|<\epsilon$. 
The three following results were obtained in [13].

Lemma 2.15. If $\bar{f}$ is differentiable at $t \in[a, b) \cap \mathbb{T}$, then $f$ is $\Delta$-differentiable at $t$ and $f^{\Delta}(t)=\bar{f}^{\prime}(t)$.

Theorem 2.16. Consider a function $f: \mathbb{T} \rightarrow \mathbb{R}$ and its extension $\bar{f}:[a, b] \rightarrow \mathbb{R}$. Then, $f$ is absolutely continuous on $\mathbb{T}$ if and only if $\bar{f}$ is absolutely continuous on $[a, b]$.

Theorem 2.17. A function $f: \mathbb{T} \rightarrow \mathbb{R}$ is absolutely continuous on $\mathbb{T}$ if and only if $f$ is $\Delta$ differentiable $\Delta$-almost everywhere on $\mathbb{T}_{0}, f^{\Delta} \in L_{\Delta}^{1}\left(\mathbb{T}_{0}\right)$ and

$$
\int_{[a, t) \cap \mathbb{T}} f^{\Delta}(s) \Delta s=f(t)-f(a), \quad \text { for every } t \in \mathbb{T} .
$$

We also recall the Banach Lemma.

Lemma 2.18. Let $E$ be a Banach space and $u:[a, b] \rightarrow E$ an absolutely continuous function, then the measure of the set $\left\{t \in[a, b]: u(t)=0\right.$ and $\left.u^{\prime}(t) \neq 0\right\}$ is zero.

Using the previous results, we now prove two propositions that will be used later.

Proposition 2.19. Let $g \in L_{\Delta}^{1}\left(\mathbb{T}_{0}\right)$ and $G: \mathbb{T} \rightarrow \mathbb{R}$ the function defined by

$$
G(t):=\int_{[a, t) \cap \mathbb{T}} g(s) \Delta s
$$

Then, $G^{\Delta}(t)=g(t) \Delta$-almost everywhere on $\mathbb{T}_{0}$.

Proof. By Theorem 2.13, remark that

$$
G(t)=\int_{[a, t)} \widehat{g}(s) d s \quad \text { for every } t \in \mathbb{T} \text {. }
$$

We can also check that for $t_{i}$ right scattered,

$$
\bar{G}(t)=\int_{\left[a, t_{i}\right)} \widehat{g}(s) d s+\int_{\left[t_{i}, t\right)} \widehat{g}(s) d s \quad \text { if } t \in\left(t_{i}, \sigma\left(t_{i}\right)\right) .
$$

Obviously,

$$
\bar{G}(t)=\int_{[a, t)} \widehat{g}(s) d s \quad \text { for every } t \in[a, b]
$$


It is well known that $\bar{G}^{\prime}(t)=\widehat{g}(t)$ almost everywhere on $[a, b]$. By Lemma 2.15, we have that $G^{\Delta}(t)=\bar{G}^{\prime}(t)=\widehat{g}(t)=g(t)$ except on a set $A \subset \mathbb{T}_{0}$ such that $\mu_{L}(A)=0$. Since $\bar{G}$ is continuous, for $t_{i} \in R_{\mathbb{T}} \cap \mathbb{T}_{0}$ right scattered,

$$
\bar{G}^{\Delta}\left(t_{i}\right)=\frac{G\left(\sigma\left(t_{i}\right)\right)-G\left(t_{i}\right)}{\mu\left(t_{i}\right)}=g\left(t_{i}\right)
$$

by Theorem 2.2(ii). Then, $G$ is $\Delta$-differentiable at $t \in\left(\mathbb{T}_{0} \backslash A\right) \cup R_{\mathbb{T}} \cap \mathbb{T}_{0}$. By Proposition 2.12(ii), $\mu_{\Delta}\left(A \backslash\left(R_{\mathbb{T}} \cap \mathbb{T}_{0}\right)\right)=0$ and, then, the proposition is proved.

Proposition 2.20. Let $u: \mathbb{T} \rightarrow \mathbb{R}$ be an absolutely continuous function, then the $\Delta$-measure of the set $\left\{t \in \mathbb{T}_{0} \backslash R_{\mathbb{T}_{0}}: u(t)=0\right.$ and $\left.u^{\Delta}(t) \neq 0\right\}$ is zero.

Proof. It suffices to consider the extension $\bar{u}$ of $u$ on $[a, b]$ and successively apply Theorem 2.16, Lemmas 2.18, 2.15, and the Proposition 2.12(ii).

We recall a notion of Sobolev's space of functions defined on a bounded time scale $\mathbb{T}$ where $a=\min \mathbb{T}<\max \mathbb{T}=b$. The definition and the result are from [14].

Definition 2.21. We say that a function $u: \mathbb{T} \rightarrow \mathbb{R}$ belongs to $W_{\Delta}^{1,1}(\mathbb{T})$ if and only if $u \in L_{\Delta}^{1}\left(\mathbb{T}_{0}\right)$ and there exists a function $g: \mathbb{T}^{\mathcal{K}} \rightarrow \mathbb{R}$ such that $g \in L_{\Delta}^{1}\left(\mathbb{T}_{0}\right)$ and

$$
\int_{\mathbb{T}_{0}} u(s) \phi^{\Delta}(s) \Delta s=-\int_{\mathbb{T}_{0}} g(s) \phi(\sigma(s)) \Delta s \quad \text { for every } \phi \in \mathrm{C}_{0, \mathrm{rd}}^{1}(\mathbb{T})
$$

with

$$
C_{0, \mathrm{rd}}^{1}(\mathbb{T}):=\left\{f: \mathbb{T} \longrightarrow \mathbb{R}: f \in C_{\mathrm{rd}}^{1}(\mathbb{T}), f(a)=0=f(b)\right\}
$$

We say that a function $f: \mathbb{T} \rightarrow \mathbb{R}^{n}$ is in the set $W_{\Delta}^{1,1}\left(\mathbb{T}, \mathbb{R}^{n}\right)$ if each of its components $f_{i}$ are in $W_{\Delta}^{1,1}(\mathbb{T})$.

Theorem 2.22. Suppose that $u \in W_{\Delta}^{1,1}(\mathbb{T})$ and that (2.22) holds for a function $g \in L_{\Delta}^{1}\left(\mathbb{T}_{0}\right)$. Then, there exists a unique function $x: \mathbb{T} \rightarrow \mathbb{R}$ absolutely continuous such that $\Delta$-almost everywhere on $\mathbb{T}_{0}$, one has $x=u$ and $x^{\Delta}=g$. Moreover, if $g$ is $r d$-continuous on $\mathbb{T}_{0}$, then there exists a unique function $x \in C_{r d}^{1}(\mathbb{T})$ such that $x=u \Delta$-almost everywhere on $\mathbb{T}_{0}$ and such that $x^{\Delta}=g$ on $\mathbb{T}_{0}$.

By the previous theorem, we can conclude that $u \in W_{\Delta}^{1,1}(\mathbb{T})$ is also continuous.

Remark 2.23. If $x \in W_{\Delta}^{1,1}\left(\mathbb{T}, \mathbb{R}^{n}\right)$, then its components $x_{i}$ are in $W_{\Delta}^{1,1}(\mathbb{T})$. By Theorems 2.22 and 2.17, $x$ is $\Delta$-differentiable $\Delta$-a.e. on $\mathbb{T}$. From Example 2.5, we obtain $\|x(t)\|^{\Delta}=$ $\left\langle x(t), x^{\Delta}(t)\right\rangle /\|x(t)\| \Delta$-a.e. on $\{t \in \mathbb{T}: t=\sigma(t)\}$.

We prove two maximum principles that will be useful to get a priori bounds for solutions of systems considered in this paper. 
Lemma 2.24. Let $r \in W_{\Delta}^{1,1}(\mathbb{T})$ such that $r^{\Delta}(t)<0 \Delta$-a.e. $t \in\left\{t \in \mathbb{T}_{0}: r(\sigma(t))>0\right\}$. If one of the following conditions holds,

(i) $r(a) \leq 0$,

(ii) $r(a) \leq r(b)$,

then $r(t) \leq 0$, for every $t \in \mathbb{T}$.

Proof. Suppose the conclusion is false. Then, there exists $t_{0} \in \mathbb{T}$ such that $r\left(t_{0}\right)=\max _{t \in \mathbb{T}} r(t)>$ 0 , since $r$ is continuous on $\mathbb{T}$. If $t_{0}>\rho\left(t_{0}\right)$, then $r^{\Delta}\left(\rho\left(t_{0}\right)\right)$ exists, since $\mu_{\Delta}\left(\left\{\rho\left(t_{0}\right)\right\}\right)=t_{0}-\rho\left(t_{0}\right)>0$ and because $r \in W_{\Delta}^{1,1}(\mathbb{T})$. Then,

$$
r^{\Delta}\left(\rho\left(t_{0}\right)\right)=\frac{r\left(t_{0}\right)-r\left(\rho\left(t_{0}\right)\right)}{t_{0}-\rho\left(t_{0}\right)} \geq 0
$$

which is a contradiction since $r\left(t_{0}\right)=r\left(\sigma\left(\rho\left(t_{0}\right)\right)\right)>0$. If $t_{0}=\rho\left(t_{0}\right)>a$, then there exists an interval $\left[t_{1}, \rho\left(t_{0}\right)\right)$ such that $r(\sigma(t))>0$ for all $t \in\left[t_{1}, \rho\left(t_{0}\right)\right) \cap \mathbb{T}$. Thus,

$$
0 \leq r\left(t_{0}\right)-r\left(t_{1}\right)=r\left(\rho\left(t_{0}\right)\right)-r\left(t_{1}\right)=\int_{\left[t_{1}, \rho\left(t_{0}\right)\right) \cap \mathbb{T}} r^{\Delta}(s) \Delta s<0
$$

by hypothesis and by Theorem 2.17. Hence, we get a contradiction. The case $t_{0}=a$ is impossible if hypothesis (i) holds and if $r(a) \leq r(b)$, we must have $r(a)=r(b)$. If we take $t_{0}=b$, by using previous steps of this proof, one can check that $r(b) \leq 0$ and, then, the lemma is proved.

Lemma 2.25. Let $r \in W_{\Delta}^{1,1}(\mathbb{T})$ be a function such that $r^{\Delta}(t)>0 \Delta$-a.e. on $\left\{t \in \mathbb{T}^{k}: r(t)>0\right\}$ if $r(a) \geq r(b)$, then $r(t) \leq 0$, for every $t \in \mathbb{T}$.

Proof. If there exists $t \in \mathbb{T}$ such that $r(t)>0$, then there exists a $t_{0} \in \mathbb{T}$ such that $r\left(t_{0}\right)=$ $\max _{t \in \mathbb{T}} r(t)>0$. If $t_{0}<b$ and $t_{0}<\sigma\left(t_{0}\right)$, then $\mu_{\Delta}\left(\left\{t_{0}\right\}\right)=\sigma\left(t_{0}\right)-t_{0}>0$. Since $r \in W_{\Delta}^{1,1}(\mathbb{T}), r^{\Delta}$ exists $\Delta$-almost everywhere. Then, we must have

$$
r^{\Delta}\left(t_{0}\right)=\frac{r\left(\sigma\left(t_{0}\right)\right)-r\left(t_{0}\right)}{\sigma\left(t_{0}\right)-t_{0}} \leq 0
$$

which contradicts the hypothesis of the lemma. If $t_{0}<b$ and $t_{0}=\sigma\left(t_{0}\right)$, there exists an interval $\left[t_{0}, t_{1}\right)$ such that $r(t)>0$ for every $t \in\left[t_{0}, t_{1}\right) \cap \mathbb{T}$. Then,

$$
0<\int_{\left[t_{0}, t_{1}\right) \cap \mathbb{T}} r^{\Delta}(s) \Delta s=r\left(t_{1}\right)-r\left(t_{0}\right)
$$

by Theorem 2.17, which contradicts the fact that $r\left(t_{0}\right)$ is a maximum. If $t_{0}=b$, then by hypothesis, we must have $r(a)=r(b)$. Thus, we can take $t_{0}=a$, and by using the previous steps of this proof, one can check that $r(a) \leq 0$. Then, the lemma is proved. 
Definition 2.26. For $\epsilon>0$, the exponential function $e_{\epsilon}\left(\cdot, t_{0}\right): \mathbb{T} \rightarrow \mathbb{R}$ may be defined as the unique solution of the initial value problem

$$
x^{\Delta}(t)=\epsilon x(t), \quad x\left(t_{0}\right)=1 .
$$

More explicitly, the exponential function $e_{\epsilon}\left(\cdot, t_{0}\right)$ is given by the formula

$$
e_{\epsilon}\left(t, t_{0}\right)=\exp \left(\int_{t_{0}}^{t} \xi_{\epsilon}(\mu(s)) \Delta s\right)
$$

where for $h \geq 0$, we define $\xi_{\epsilon}(h)$ as

$$
\xi_{\epsilon}(h)= \begin{cases}\epsilon, & \text { if } h=0 \\ \frac{\log (1+h \epsilon)}{h}, & \text { otherwise. }\end{cases}
$$

As direct consequences of Proposition 2.19 and Theorem 2.3, we get the following results.

Proposition 2.27. If $g \in L_{\Delta}^{1}\left(\mathbb{T}_{0}, \mathbb{R}^{n}\right)$, the function $x: \mathbb{T} \rightarrow \mathbb{R}^{n}$ defined by

$$
x(t)=e_{1}(a, t)\left(x_{0}+\int_{[a, t) \cap \mathbb{T}} e_{1}(s, a) g(s) \Delta s\right)
$$

is a solution of the problem

$$
\begin{gathered}
x^{\Delta}(t)+x(\sigma(t))=g(t), \quad \Delta \text {-a.e. } t \in \mathbb{T}_{0}, \\
x(a)=x_{0} .
\end{gathered}
$$

Proposition 2.28. If $g \in L_{\Delta}^{1}\left(\mathbb{T}_{0}, \mathbb{R}^{n}\right)$, then the function $x: \mathbb{T} \rightarrow \mathbb{R}^{n}$ defined by

$$
x(t)=\frac{1}{e_{1}(t, a)}\left(\frac{1}{e_{1}(b, a)-1} \int_{[a, b) \cap \mathbb{T}} g(s) e_{1}(s, a) \Delta s+\int_{[a, t) \cap \mathbb{T}} g(s) e_{1}(s, a) \Delta s\right)
$$

is a solution of the problem

$$
\begin{gathered}
x^{\Delta}(t)+x(\sigma(t))=g(t), \quad \Delta \text {-a.e. } t \in \mathbb{T}_{0}, \\
x(a)=x(b) .
\end{gathered}
$$


Proposition 2.29. If $g \in L_{\Delta}^{1}\left(\mathbb{T}_{0}, \mathbb{R}^{n}\right)$, then the function $x: \mathbb{T} \rightarrow \mathbb{R}^{n}$ defined by

$$
x(t)=e_{1}(t, a)\left(\frac{e_{1}(b, a)}{1-e_{1}(b, a)} \int_{[a, b) \cap \mathbb{T}} \frac{g(s)}{e_{1}(\sigma(s), a)} \Delta s+\int_{[a, t) \cap \mathbb{T}} \frac{g(s)}{e_{1}(\sigma(s), a)} \Delta s\right)
$$

is a solution of the problem

$$
\begin{gathered}
x^{\Delta}(t)-x(t)=g(t), \quad \Delta \text {-a.e. } t \in \mathbb{T}_{0}, \\
x(a)=x(b) .
\end{gathered}
$$

We now define a notion of Carathéodory functions on a compact time scale.

Definition 2.30. A function $f: \mathbb{T}_{0} \times \mathbb{R}^{n} \rightarrow \mathbb{R}^{n}$ is called a $\Delta$-Carathéodory function if the three following conditions hold.

(C-i) The map $t \mapsto f(t, x)$ is $\Delta$-measurable for every $x \in \mathbb{R}^{n}$.

(C-ii) The map $x \mapsto f(t, x)$ is continuous $\Delta$-a.e. $t \in \mathbb{T}_{0}$.

(C-iii) For every $r>0$, there exists a function $h_{r} \in L_{\Delta}^{1}\left(\mathbb{T}_{0},[0, \infty)\right)$ such that $\|f(t, x)\| \leq$ $h_{r}(t) \Delta$-a.e. $t \in \mathbb{T}_{0}$ and for every $x \in \mathbb{R}^{n}$ such that $\|x\| \leq R$.

\section{Existence Theorem for the Problem (1.1)}

In this section, we establish an existence result for the problem (1.1). A solution of this problem will be a function $x \in W_{\Delta}^{1,1}\left(\mathbb{T}, \mathbb{R}^{n}\right)$ satisfying (1.1). Let us recall that $\mathbb{T}$ is compact and $a=\min \mathbb{T}<\max \mathbb{T}=b$. We introduce the notion of solution tube for the problem (1.1).

Definition 3.1. Let $(v, M) \in W_{\Delta}^{1,1}\left(\mathbb{T}, \mathbb{R}^{n}\right) \times W_{\Delta}^{1,1}(\mathbb{T},[0, \infty))$. We say that $(v, M)$ is a solution tube of (1.1) if

(i) $\left\langle x-v(\sigma(t)), f(t, x)-v^{\Delta}(t)\right\rangle \leq M(\sigma(t)) M^{\Delta}(t) \Delta$-a.e. $t \in \mathbb{T}_{0}$ and for every $x \in \mathbb{R}^{n}$ such that $\|x-v(\sigma(t))\|=M(\sigma(t))$,

(ii) $v^{\Delta}(t)=f(t, v(\sigma(t))) \Delta$-a.e. $t \in \mathbb{T}_{0}$ such that $M(\sigma(t))=0$,

(iii) $M(t)=0$ for every $t \in \mathbb{T}_{0}$ such that $M(\sigma(t))=0$,

(iv) If (BC) denotes (1.3), $\left\|x_{0}-v(a)\right\| \leq M(a)$; if (BC) denotes (1.4), then $\|v(b)-v(a)\| \leq$ $M(a)-M(b)$.

We denote

$$
T(v, M)=\left\{x \in W_{\Delta}^{1,1}\left(\mathbb{T}, \mathbb{R}^{n}\right):\|x(t)-v(t)\| \leq M(t) \text { for every } t \in \mathbb{T}\right\}
$$

If $\mathbb{T}$ is a real interval $[a, b]$, our definition of solution tube is equivalent to the notion of solution tube introduced in [5]. 
We consider the following problem.

$$
\begin{gathered}
x^{\Delta}(t)+x(\sigma(t))=f(t, \tilde{x}(\sigma(t)))+\tilde{x}(\sigma(t)), \quad \Delta \text {-a.e. } t \in \mathbb{T}_{0}, \\
x \in(B C),
\end{gathered}
$$

where

$$
\tilde{x}(s)= \begin{cases}\frac{M(s)}{\|x-v(s)\|}(x-v(s))+v(s) & \text { if }\|x-v(s)\|>M(s), \\ x & \text { otherwise. }\end{cases}
$$

Let us define the operator $T_{I}: C\left(\mathbb{T}, \mathbb{R}^{n}\right) \rightarrow C\left(\mathbb{T}, \mathbb{R}^{n}\right)$ by

$$
T_{I}(x)(t)=e_{1}(a, t)\left(x_{0}+\int_{[a, t) \cap \mathbb{T}} e_{1}(s, a)(f(s, \tilde{x}(\sigma(s)))+\tilde{x}(\sigma(s))) \Delta s\right) .
$$

Proposition 3.2. If $(v, M) \in W_{\Delta}^{1,1}\left(\mathbb{T}, \mathbb{R}^{n}\right) \times W_{\Delta}^{1,1}(\mathbb{T},[0, \infty))$ is a solution tube of (1.1), (1.3), then $T_{I}: C\left(\mathbb{T}, \mathbb{R}^{n}\right) \rightarrow C\left(\mathbb{T}, \mathbb{R}^{n}\right)$ is compact.

Proof. We first observe that from Definitions 2.30 and 3.1, there exists a function $h \in$ $L_{\Delta}^{1}\left(\mathbb{T}_{0},[0, \infty)\right)$ such that $\|f(t, \tilde{x}(\sigma(t)))+\tilde{x}(\sigma(t))\| \leq h(t) \Delta$-a.e. $t \in \mathbb{T}_{0}$ for every $x \in C\left(\mathbb{T}, \mathbb{R}^{n}\right)$.

Let $\left\{x_{n}\right\}_{n \in \mathbb{N}}$ be a sequence of $C\left(\mathbb{T}, \mathbb{R}^{n}\right)$ converging to $x \in C\left(\mathbb{T}, \mathbb{R}^{n}\right)$. By Proposition 2.9,

$$
\begin{aligned}
& \left\|T_{I}\left(x_{n}(t)\right)-T_{I}(x(t))\right\| \\
& \quad=\left\|\int_{[a, t) \cap \mathbb{T}} e_{1}(s, t)\left(\left(f\left(s, \widetilde{x_{n}}(\sigma(s))\right)+\widetilde{x_{n}}(\sigma(s))\right)-(f(s, \tilde{x}(\sigma(s)))+\tilde{x}(\sigma(s)))\right) \Delta s\right\| \\
& \quad \leq K\left(\int_{[a, b) \cap \mathbb{T}}\left\|f\left(s, \widetilde{x_{n}}(\sigma(s))\right)+\widetilde{x_{n}}(\sigma(s))-(f(s, \tilde{x}(\sigma(s)))+\tilde{x}(\sigma(s)))\right\| \Delta s\right),
\end{aligned}
$$

where $K:=\max _{t, t_{1} \in \mathbb{T}}\left|e_{1}\left(t_{1}, t\right)\right|$.

Then, we must show that the sequence $\left\{g_{n}\right\}_{n \in \mathbb{N}}$ defined by

$$
g_{n}(s):=f\left(s, \widetilde{x_{n}}(\sigma(s))\right)+\widetilde{x_{n}}(\sigma(s))
$$

converges to the function $g$ in $L_{\Delta}^{1}\left(\mathbb{T}_{0}, \mathbb{R}^{n}\right)$ where

$$
g(s)=f(s, \tilde{x}(\sigma(s)))+\tilde{x}(\sigma(s)) .
$$

We can easily check that $\tilde{x}_{n}(t) \rightarrow \tilde{x}(t)$ for every $t \in \mathbb{T}_{0}$ and, then, by (C-ii) of Definition 2.30, $g_{n}(s) \rightarrow g(s) \Delta$-a.e. $s \in \mathbb{T}_{0}$. Using also the fact that $\left\|g_{n}(s)\right\| \leq h(s) \Delta$-a.e. $s \in \mathbb{T}_{0}$, we deduce that $g_{n} \rightarrow g$ in $L_{\Delta}^{1}\left(\mathbb{T}_{0}, \mathbb{R}^{n}\right)$ by Theorem 2.10 . This prove the continuity of $T_{I}$. 
For the second part of the proof, we have to show that the set $T_{I}\left(C\left(\mathbb{T}, \mathbb{R}^{n}\right)\right)$ is relatively compact. Let $y=T_{I}(x) \in T_{I}\left(C\left(\mathbb{T}, \mathbb{R}^{n}\right)\right)$. Therefore,

$$
\begin{aligned}
\left\|T_{I}(x(t))\right\| & \leq K\left(\left\|x_{0}\right\|+\int_{[a, b) \cap \mathbb{T}}\|f(s, \tilde{x}(\sigma(s)))+\widetilde{x}(\sigma(s))\| \Delta s\right) \\
& \leq K\left(\left\|x_{0}\right\|+\|h\|_{L_{\Delta}^{1}\left(\mathbb{T}_{0}\right)}\right) .
\end{aligned}
$$
$t_{1}, t_{2} \in \mathbb{T}$,

So, $T_{I}\left(C\left(\mathbb{T}, \mathbb{R}^{n}\right)\right)$ is uniformly bounded. This set is also equicontinuous since for every

$$
\left\|T_{I}\left(x\left(t_{2}\right)\right)-T_{I}\left(x\left(t_{1}\right)\right)\right\| \leq K \int_{\left[t_{1}, t_{2}\right) \cap \mathbb{T}} h(s) \Delta s
$$

By an analogous version of the Arzelà-Ascoli Theorem adapted to our context, $T_{I}\left(C\left(\mathbb{T}, \mathbb{R}^{n}\right)\right)$ is relatively compact. Hence, $T_{I}$ is compact.

We now define the operator $T_{P}: C\left(\mathbb{T}, \mathbb{R}^{n}\right) \rightarrow C\left(\mathbb{T}, \mathbb{R}^{n}\right)$ by

$$
\begin{aligned}
T_{P}(x)(t)=\frac{1}{e_{1}(t, a)}( & \frac{1}{e_{1}(b, a)-1} \int_{[a, b) \cap \mathbb{T}}(f(s, \tilde{x}(\sigma(s)))+\tilde{x}(\sigma(s))) e_{1}(s, a) \Delta s \\
& \left.+\int_{[a, t) \cap \mathbb{T}}(f(s, \tilde{x}(\sigma(s)))+\tilde{x}(\sigma(s))) e_{1}(s, a) \Delta s\right) .
\end{aligned}
$$

The following result can be proved as the previous one.

Proposition 3.3. If $(v, M) \in W_{\Delta}^{1,1}\left(\mathbb{T}, \mathbb{R}^{n}\right) \times W_{\Delta}^{1,1}(\mathbb{T},[0, \infty))$ is a solution tube of (1.1), (1.4), then the operator $T_{P}: C\left(\mathbb{T}, \mathbb{R}^{n}\right) \rightarrow C\left(\mathbb{T}, \mathbb{R}^{n}\right)$ is compact.

Now, we can obtain the main theorem of this section.

Theorem 3.4. If $(v, M) \in W_{\Delta}^{1,1}\left(\mathbb{T}, \mathbb{R}^{n}\right) \times W_{\Delta}^{1,1}(\mathbb{T},[0, \infty))$ is a solution tube of $(1.1)$, then the problem (1.1) has a solution $x \in W_{\Delta}^{1,1}\left(\mathbb{T}, \mathbb{R}^{n}\right) \cap T(v, M)$.

Proof. By Proposition 3.2 (resp., Proposition 3.3), $T_{I}$ (resp., $T_{P}$ ) is compact. It has a fixed point by the Schauder fixed-point Theorem. Proposition 2.27 (resp., Proposition 2.28) implies that this fixed point is a solution for the problem (3.2). Then, it suffices to show that for every solution $x$ of $(3.2), x \in T(v, M)$. 
Consider the set $A=\left\{t \in \mathbb{T}_{0}:\|x(\sigma(t))-v(\sigma(t))\|>M(\sigma(t))\right\}$. By Remark 2.23, $\Delta$-a.e. on the set $\tilde{A}=\{t \in A: t=\sigma(t)\}$, we have

$$
\begin{aligned}
(\|x(t)-v(t)\|-M(t))^{\Delta} & =\frac{\left\langle x(t)-v(t), x^{\Delta}(t)-v^{\Delta}(t)\right\rangle}{\|x(t)-v(t)\|}-M^{\Delta}(t) \\
& =\frac{\left\langle x(\sigma(t))-v(\sigma(t)), x^{\Delta}(t)-v^{\Delta}(t)\right\rangle}{\|x(\sigma(t))-v(\sigma(t))\|}-M^{\Delta}(t) .
\end{aligned}
$$

If $t \in A$ is right scattered, then $\mu_{\Delta}(\{t\})>0$ and

$$
\begin{aligned}
(\| x(t) & -v(t) \|-M(t))^{\Delta} \\
& =\frac{\|x(\sigma(t))-v(\sigma(t))\|-\|x(t)-v(t)\|}{\mu(t)}-M^{\Delta}(t) \\
& =\frac{\|x(\sigma(t))-v(\sigma(t))\|^{2}-\|x(\sigma(t))-v(\sigma(t))\|\|x(t)-v(t)\|}{\mu(t)\|x(\sigma(t))-v(\sigma(t))\|}-M^{\Delta}(t) \\
& \leq \frac{\langle x(\sigma(t))-v(\sigma(t)), x(\sigma(t))-v(\sigma(t))-(x(t)-v(t))\rangle}{\mu(t)\|x(\sigma(t))-v(\sigma(t))\|}-M^{\Delta}(t) \\
& =\frac{\left\langle x(\sigma(t))-v(\sigma(t)), x^{\Delta}(t)-v^{\Delta}(t)\right\rangle}{\|x(\sigma(t))-v(\sigma(t))\|}-M^{\Delta}(t) .
\end{aligned}
$$

Therefore, since $(v, M)$ is a solution tube of (1.1), we have $\Delta$-a.e. on $\{t \in A: M(\sigma(t))>$ $0\}$ that

$$
\begin{aligned}
(\|x(t)-v(t)\|-M(t))^{\Delta} & \\
\leq & \frac{\left\langle x(\sigma(t))-v(\sigma(t)), f(t, \tilde{x}(\sigma(t)))+(\tilde{x}(\sigma(t))-x(\sigma(t)))-v^{\Delta}(t)\right\rangle}{\|x(\sigma(t))-v(\sigma(t))\|}-M^{\Delta}(t) \\
= & \frac{\left\langle\tilde{x}(\sigma(t))-v(\sigma(t)), f(t, \tilde{x}(\sigma(t)))-v^{\Delta}(t)\right\rangle}{M(\sigma(t))} \\
& +(M(\sigma(t))-\|x(\sigma(t))-v(\sigma(t))\|)-M^{\Delta}(t) \\
< & \frac{M(\sigma(t)) M^{\Delta}(t)}{M(\sigma(t))}-M^{\Delta}(t)=0 .
\end{aligned}
$$


On the other hand, we have $\Delta$-a.e. on $\{t \in A: M(\sigma(t))=0\}$ that

$$
\begin{aligned}
(\| x(t) & -v(t) \|-M(t))^{\Delta} \\
\leq & \frac{\left\langle x(\sigma(t))-v(\sigma(t)), f(t, \tilde{x}(\sigma(t)))+(\tilde{x}(\sigma(t))-x(\sigma(t)))-v^{\Delta}(t)\right\rangle}{\|x(\sigma(t))-v(\sigma(t))\|}-M^{\Delta}(t) \\
= & \frac{\left\langle x(\sigma(t))-v(\sigma(t)), f(t, v(\sigma(t)))-v^{\Delta}(t)\right\rangle}{\|x(\sigma(t))-v(\sigma(t))\|} \\
& -(\|x(\sigma(t))-v(\sigma(t))\|)-M^{\Delta}(t) \\
< & -M^{\Delta}(t)=0 .
\end{aligned}
$$

This last equality follows from Definition 3.1(iii) and Proposition 2.20.

If we set $r(t):=\|x(t)-v(t)\|-M(t)$, then $r^{\Delta}(t)<0 \Delta$-almost everywhere on $A=$ $\left\{t \in \mathbb{T}_{0}: r(\sigma(t))>0\right\}$. Moreover, since $(v, M)$ is a solution tube of (1.1) and $x$ satisfies (1.3) (resp., $x$ satisfies (1.4)), then $r(a) \leq 0$ (resp., $r(a)-r(b) \leq\|v(a)-v(b)\|-(M(a)-M(b)) \leq 0$ ). Lemma 2.24 implies that $A=\emptyset$. Therefore, $x \in T(v, M)$ and, hence, the theorem is proved.

Existence theorems are obtained in [7] for the problem (1.1), (1.3) when $f: \mathbb{T} \times \mathbb{R}^{n} \rightarrow$ $\mathbb{R}^{n}$ is continuous by using a hypothesis different of ours. When $f$ is bounded, we can directly use the Schauder fixed-point Theorem to deduce the existence of a solution to (1.1), (1.3). We now show that in the case where $f$ is unbounded, Theorems 4.7 and 4.8 of [7] become corollaries of our existence theorem.

Corollary 3.5. Let $f: \mathbb{T}^{\kappa} \times \mathbb{R}^{n} \rightarrow \mathbb{R}^{n}$ be an unbounded continuous function. If there exist nonnegative constants $L$ and $N$ such that

$$
\|f(t, p)\| \leq-2 L\langle p, f(t, p)\rangle+N
$$

for every $t \in \mathbb{T}^{\kappa}$ and every $p \in \mathbb{R}^{n}$, then the problem (1.1), (1.3) has at least one solution.

Proof. Observe that $L>0$ since $f$ is unbounded. By hypothesis, there exists a constant $K:=$ $N / 2 L$ such that $\langle p, f(t, p)\rangle \leq K$. Let us define $M: \mathbb{T} \rightarrow[0, \infty)$ by

$$
M(t):=\left\|x_{0}\right\|+1+\int_{[a, t) \cap \mathbb{T}} K \Delta s
$$

Then, $M^{\Delta}(t)=K$ for every $t \in \mathbb{T}$ and, thus,

$$
\langle p, f(t, p)\rangle \leq K \leq M^{\Delta}(t) M(\sigma(t))
$$

for every $t \in \mathbb{T}$ and every $p \in \mathbb{R}^{n}$. Then, if we take $v \equiv 0$, we get a solution tube $(v, M)$ for our problem and by Theorem 3.4, the problem has a solution $x$ such that $\|x(t)\| \leq\left\|x_{0}\right\|+1+K(t-a)$ for every $t \in \mathbb{T}$. 
Corollary 3.6. Let $f: \mathbb{T}^{\kappa} \times \mathbb{R}^{n} \rightarrow \mathbb{R}^{n}$ be an unbounded continuous function. If there exists a nonnegative constant $K$ such that

$$
\langle p, f(t, p)\rangle \leq K
$$

for every $t \in \mathbb{T}^{\kappa}$ and every $p \in \mathbb{R}^{n}$, then the problem (1.1), (1.3) has at least one solution.

\section{Existence Theorem for the Problem (1.2)}

In this section, we establish an existence result for the problem (1.2). A solution of this problem will be a function $x \in W_{\Delta}^{1,1}\left(\mathbb{T}, \mathbb{R}^{n}\right)$ for which (1.2) is satisfied. As before, $\mathbb{T}$ is compact and $a=\min \mathbb{T}<\max \mathbb{T}=b$. We introduce the notion of solution tube for the problem (1.2). Conditions of this definition are slightly different than conditions in Definition 3.1.

Definition 4.1. Let $(v, M) \in W_{\Delta}^{1,1}\left(\mathbb{T}, \mathbb{R}^{n}\right) \times W_{\Delta}^{1,1}(\mathbb{T},[0, \infty))$. We say that $(v, M)$ is a solution tube of (1.2) if

(i) $\left\langle x-v(t), f(t, x)-v^{\Delta}(t)\right\rangle \geq M(t) M^{\Delta}(t) \Delta$-a.e. $t \in \mathbb{T}_{0}$ and for every $x \in \mathbb{R}^{n}$ such that $\|x-v(t)\|=M(t)$

(ii) $v^{\Delta}(t)=f(t, v(t))$ and $M^{\Delta}(t)=0, \Delta$-a.e. $t \in \mathbb{T}_{0}$ such that $M(t)=0$,

(iii) $\|v(b)-v(a)\| \leq M(b)-M(a)$.

We consider the following problem.

$$
\begin{gathered}
x^{\Delta}(t)-x(t)=f(t, \tilde{x}(t))-\tilde{x}(t), \quad \Delta \text {-a.e. } t \in \mathbb{T}_{0}, \\
x(a)=x(b) .
\end{gathered}
$$

where $\tilde{x}(t)$ is defined in (3.3).

Let us define the operator $T_{P *}: C\left(\mathbb{T}, \mathbb{R}^{n}\right) \rightarrow C\left(\mathbb{T}, \mathbb{R}^{n}\right)$ by

$$
\begin{aligned}
T_{P *}(x)(t)=e_{1}(t, a) & \left(\frac{e_{1}(b, a)}{1-e_{1}(b, a)} \int_{[a, b) \cap \mathbb{T}} \frac{(f(s, \tilde{x}(s))-\tilde{x}(s))}{e_{1}(\sigma(s), a)} \Delta s\right. \\
& \left.+\int_{[a, t) \cap \mathbb{T}} \frac{(f(s, \tilde{x}(s))-\tilde{x}(s))}{e_{1}(\sigma(s), a)} \Delta s\right) .
\end{aligned}
$$

The following result can be proved as Proposition 3.2.

Proposition 4.2. If $(v, M) \in W_{\Delta}^{1,1}\left(\mathbb{T}, \mathbb{R}^{n}\right) \times W_{\Delta}^{1,1}(\mathbb{T},[0, \infty))$ is a solution tube of $(1.2)$, then the operator $T_{P_{*}}: C\left(\mathbb{T}, \mathbb{R}^{n}\right) \rightarrow C\left(\mathbb{T}, \mathbb{R}^{n}\right)$ is compact.

Here is the main existence theorem for problem (1.2).

Theorem 4.3. If $(v, M) \in W_{\Delta}^{1,1}\left(\mathbb{T}, \mathbb{R}^{n}\right) \times W_{\Delta}^{1,1}(\mathbb{T},[0, \infty))$ is a solution tube of $(1.2)$, then the problem (1.2) has a solution $x \in W_{\Delta}^{1,1}\left(\mathbb{T}, \mathbb{R}^{n}\right) \cap T(v, M)$. 
Proof. By Proposition 4.2, $T_{P *}$ is compact. Then, by the Schauder fixed-point Theorem, $T_{P *}$ has a fixed point which is a solution of (4.1) by Proposition 2.29. It suffices to show that for every solution $x$ of $(4.1), x \in T(v, M)$.

Let us consider the set $A=\left\{t \in \mathbb{T}_{0}:\|x(t)-v(t)\|>M(t)\right\}$. By Remark 2.23, $\Delta$-a.e. on the set $\widetilde{A}=\{t \in A: t=\sigma(t)\}$ we have

$$
(\|x(t)-v(t)\|-M(t))^{\Delta}=\frac{\left\langle x(t)-v(t), x^{\Delta}(t)-v^{\Delta}(t)\right\rangle}{\|x(t)-v(t)\|}-M^{\Delta}(t) .
$$

If $t \in A$ is right scattered, then $\mu_{\Delta}(\{t\})>0$ and

$$
\begin{aligned}
(\| x(t) & -v(t) \|-M(t))^{\Delta} \\
& =\frac{\|x(\sigma(t))-v(\sigma(t))\|-\|x(t)-v(t)\|}{\mu(t)}-M^{\Delta}(t) \\
& =\frac{\|x(\sigma(t))-v(\sigma(t))\|\|x(t)-v(t)\|-\|x(t)-v(t)\|^{2}}{\mu(t)\|x(t)-v(t)\|}-M^{\Delta}(t) \\
& \geq \frac{\langle x(t)-v(t), x(\sigma(t))-v(\sigma(t))-(x(t)-v(t))\rangle}{\mu(t)\|x(t)-v(t)\|}-M^{\Delta}(t) \\
& =\frac{\left\langle x(t)-v(t), x^{\Delta}(t)-v^{\Delta}(t)\right\rangle}{\|x(t)-v(t)\|}-M^{\Delta}(t) .
\end{aligned}
$$

Since $(v, M)$ is a solution tube of $(1.2)$, we have $\Delta$-a.e. on $\{t \in A: M(t)>0\}$ that

$$
\begin{aligned}
&(\|x(t)-v(t)\|-M(t))^{\Delta} \\
& \geq \\
& \geq \frac{\left\langle x(t)-v(t), f(t, \tilde{x}(t))+(x(t)-\tilde{x}(t))-v^{\Delta}(t)\right\rangle}{\|x(t)-v(t)\|}-M^{\Delta}(t) \\
&=\frac{\left\langle\tilde{x}(t)-v(t), f(t, \tilde{x}(t))-v^{\Delta}(t)\right\rangle}{M(t)} \\
& \quad-(M(t)-\|x(t)-v(t)\|)-M^{\Delta}(t) \\
&> \frac{M(t) M^{\Delta}(t)}{M(t)}-M^{\Delta}(t)=0 .
\end{aligned}
$$


On the other hand, we have $\Delta$-a.e. on $\{t \in A: M(t)=0\}$ that

$$
\begin{aligned}
(\|x(t)-v(t)\|-M(t))^{\Delta} & \\
\geq & \frac{\left\langle x(t)-v(t), f(t, \tilde{x}(t))+(x(t)-\tilde{x}(t))-v^{\Delta}(t)\right\rangle}{\|x(t)-v(t)\|}-M^{\Delta}(t) \\
= & \frac{\left\langle x(t)-v(t), f(t, v(t))-v^{\Delta}(t)\right\rangle}{\|x(t)-v(t)\|} \\
& \quad+(\|x(t)-v(t)\|)-M^{\Delta}(t) . \\
> & 0
\end{aligned}
$$

If we set $r(t):=\|x(t)-v(t)\|-M(t)$, then $r^{\Delta}(t)>0 \Delta$-almost everywhere on $A=$ $\left\{t \in \mathbb{T}_{0}: r(t)>0\right\}$. Moreover, since $(v, M)$ is a solution tube of (1.2) and $x$ satisfies (1.4), $r(b)-r(a) \leq\|v(b)-v(a)\|-(M(b)-M(a)) \leq 0$. Lemma 2.25 implies that $A=\emptyset$. So, $x \in T(v, M)$ and the theorem is proved.

Let us observe that the following results obtained in [6] and [15], respectively, are different from ours.

Theorem 4.4. Let $f: \mathbb{T}^{\kappa} \times \mathbb{R}^{n} \rightarrow \mathbb{R}^{n}$ be a continuous function with $\mathbb{T}$ such that $\mu(t) \neq 1$. If there exist nonnegative constants $\alpha$ and $K$ such that

$$
\frac{\|f(t, p)-p\|}{h(t)} \leq 2 \alpha\langle p, f(t, p)\rangle+K
$$

for every $(t, p) \in \mathbb{T}^{\kappa} \times \mathbb{R}^{n}$, where $h: \mathbb{T} \rightarrow \mathbb{R}$ is defined by $h(t):=\exp \left(\int_{a}^{\sigma(t)} \xi_{-1}(\mu(s)) \Delta s\right)$ with

$$
\xi_{-1}(\mu(s))= \begin{cases}-1, & \text { if } \mu(s)=0, \\ \frac{\log (1-\mu(s))}{\mu(s)}, & \text { otherwise }\end{cases}
$$

then the problem (1.2) has a solution.

Theorem 4.5. Let $N \in \mathbb{N}, \mathbb{T}=\{0,1, \ldots, N, N+1\}$ and $f:\{0,1, \ldots, N\} \times \mathbb{R}^{n} \rightarrow \mathbb{R}^{n}$ a continuous function. If there exist nonnegative constants $\alpha$ and $K$ such that

$$
\frac{\|f(t, p)-p\|}{2^{t+1}} \leq 2 \alpha\langle p, f(t, p)\rangle+K
$$

for every $(t, p) \in\{0,1, \ldots, N\} \times \mathbb{R}^{n}$, then the difference equation (1.2) has one solution.

Observe that Theorem 4.3 is valid for every arbitrary time scale $\mathbb{T}$. Here is an example where (4.7) and (4.9) are not satisfied, but where Theorem 4.3 can be applied to deduce the existence of a solution. 
Example 4.6. Consider the system

$$
\begin{gathered}
x^{\Delta}(t)=-a_{1}\|x(t)\|^{2} x(t)+a_{2} x(t)-a_{3} \phi(t), \quad t \in \mathbb{T}^{\kappa}, \\
x(a)=x(b),
\end{gathered}
$$

where $a_{1}, a_{2}, a_{3}$ are real positive constants such that $-a_{1}+a_{2}-a_{3}=0$ and where $\phi: \mathbb{T}^{\kappa} \rightarrow \mathbb{R}^{n}$ is a continuous function such that $\|\phi(t)\|=1$ for every $t \in \mathbb{T}^{\kappa}$.

We first show that this system do not satisfy (4.7). Suppose there exist non-negative constants $\alpha$ and $K$ such that

$$
\frac{\left\|-a_{1}\right\| x\left\|^{2} x+a_{2} x-a_{3} \phi(t)-x\right\|}{h(t)} \leq 2 \alpha\left\langle x,-a_{1}\|x\|^{2} x+a_{2} x-a_{3} \phi(t)\right\rangle+K
$$

for every $(t, x) \in \mathbb{T}^{\kappa} \times \mathbb{R}^{n}$.

If we define $k:=\max _{t \in \mathbb{T}}\|h(t)\|$, then

$$
\begin{aligned}
\frac{-a_{1}\|x\|^{3}+a_{2}\|x\|-a_{3}-\|x\|}{k} & \leq \frac{\left\|-a_{1}\right\| x\left\|^{2} x+a_{2} x-a_{3} \phi(t)-x\right\|}{k} \\
& \leq 2 \alpha\left\langle x,-a_{1}\|x\|^{2} x+a_{2} x-a_{3} \phi(t)\right\rangle+K \\
& \leq-2 a_{1} \alpha\|x\|^{4}+2 a_{2} \alpha\|x\|^{2}+2 a_{3} \alpha\|x\|+K
\end{aligned}
$$

for every $(t, x) \in \mathbb{T}^{\kappa} \times \mathbb{R}^{n}$.

Then,

$$
2 a_{1} \alpha\|x\|^{4}+b\|x\|^{3}+c\|x\|^{2}+d\|x\|+e \leq K
$$

for every $x \in \mathbb{R}^{n}$, where $b=-a_{1} / k, c=-2 a_{2} \alpha, d=-2 a_{3} \alpha+\left(a_{2} / k\right)-(1 / k)$, and $e=-a_{3} / k$. Taking the limit as $\|x\| \rightarrow \infty$, we get a contradiction. Similarly, if $\mathbb{T}=\{0,1, \ldots, N, N+1\}$, it can be shown that (4.9) is not satisfied. On the other hand, it is easy to verify that $(v \equiv 0, M \equiv 1)$ is a solution tube of (4.10). By Theorem 4.3, this problem has a solution $x$ such that $\|x(t)\| \leq 1$ for every $t \in \mathbb{T}$.

Definition 4.1 generalizes the notions of lower and upper solutions $\alpha$ and $\beta$ introduced in [3] in the particular case where the problem (1.2) is considered with $n=1, \mathbb{T}=$ $\{0,1, \ldots, N, N+1\}$ for some $N \in \mathbb{N}$ and with $f$ depending only on $x(t)$. We recall these definitions. Consider the problem

$$
\begin{gathered}
\Delta x(t)=f(x(t)), \quad \text { for every } t \in\{0,1, \ldots, N\}, \\
x(0)=x(N+1)
\end{gathered}
$$

where $\Delta x(t)=x(t+1)-x(t)$. 
Definition 4.7. A vector $\beta=(\beta(0), \beta(1), \ldots, \beta(N+1)) \in \mathbb{R}^{(N+2)}$ (resp., $\alpha=(\alpha(0), \alpha(1), \ldots, \alpha(N+$ $1)) \in \mathbb{R}^{(N+2)}$ ) is called an upper solution (resp., a lower solution) of (4.14) if

(i) for every $t \in\{0,1, \ldots, N, N+1\}, f(\beta(t)) \geq \Delta \beta(t)$ (resp., $f(\alpha(t)) \leq \Delta \alpha(t)$ ),

(ii) $\beta(0)=\beta(N+1)$ (resp., $\alpha(0)=\alpha(N+1)$ ).

Remark that if $\alpha, \beta \in \mathbb{R}^{(N+2)}$ are, respectively, lower and upper solutions of (4.14) such that $\alpha(t) \leq \beta(t)$ for every $t \in\{0,1, \ldots, N, N+1\}$, then $((\beta+\alpha) / 2,(\beta-\alpha) / 2)$ is a solution tube for this problem. Conversely, if $(v, M)$ is a solution tube of (4.14), then $v-M$ and $v+M$ are, respectively, lower and upper solution for the same problem if, in addition, condition (ii) of Definition 4.7 is satisfied. Then, Theorem 5 of [3] becomes a corollary of Theorem 4.3.

Corollary 4.8. If $\alpha, \beta \in \mathbb{R}^{N+2}$ are, respectively, lower and upper solutions of (4.14) such that $\alpha(t) \leq$ $\beta(t)$ for every $t \in\{0,1, \ldots, N, N+1\}$, then this equation has a solution $x=(x(0), x(1), \ldots, x(N+$ $1)) \in \mathbb{R}^{(N+2)}$ such that $\alpha(t) \leq x(t) \leq \beta(t)$ for every $t \in\{0,1, \ldots, N, N+1\}$.

\section{Acknowledgment}

The author would like to thank Professor Marlene Frigon for useful discussion and comments and the FQRNT for financial support.

\section{References}

[1] V. Otero-Espinar and D. R. Vivero, "Existence of extremal solutions by approximation to a first-order initial dynamic equation with Carathéodory's conditions and discontinuous non-linearities," Journal of Difference Equations and Applications, vol. 12, no. 12, pp. 1225-1241, 2006.

[2] V. Otero-Espinar and D. R. Vivero, "The existence and approximation of extremal solutions to several first-order discontinuous dynamic equations with nonlinear boundary value conditions," Nonlinear Analysis: Theory, Methods E Applications, vol. 68, no. 7, pp. 2027-2037, 2008.

[3] C. Bereanu and J. Mawhin, "Existence and multiplicity results for periodic solutions of nonlinear difference equations," Journal of Difference Equations and Applications, vol. 12, no. 7, pp. 677-695, 2006.

[4] D. Franco, D. O'Regan, and J. Perán, "Upper and lower solution theory for first and second order difference equations," Dynamic Systems and Applications, vol. 13, no. 2, pp. 273-282, 2004.

[5] B. Mirandette, Résultats d'Existence pour des Systèmes d'Équations Différentielles du Premier Ordre avec Tube-Solution, Mémoire de Maîtrise, Université de Montréal, Montréal, Canada, 1996.

[6] Q. Dai and C. C. Tisdell, "Existence of solutions to first-order dynamic boundary value problems," International Journal of Difference Equations, vol. 1, no. 1, pp. 1-17, 2006.

[7] C. C. Tisdell and A. Zaidi, "Basic qualitative and quantitative results for solutions to nonlinear, dynamic equations on time scales with an application to economic modelling," Nonlinear Analysis: Theory, Methods \& Applications, vol. 68, no. 11, pp. 3504-3524, 2008.

[8] V. Otero-Espinar and D. R. Vivero, "Existence and approximation of extremal solutions to first-order infinite systems of functional dynamic equations," Journal of Mathematical Analysis and Applications, vol. 339, no. 1, pp. 590-597, 2008.

[9] M. Bohner and A. Peterson, Advances in Dynamic Equations on Time Scales, Birkhäuser, Boston, Mass, USA, 2003.

[10] M. Bohner and A. Peterson, Dynamic Equations on Time Scales, Birkhäuser, Boston, Mass, USA, 2001.

[11] S. Hilger, "Analysis on measure chains-a unified approach to continuous and discrete calculus," Results in Mathematics. Resultate der Mathematik, vol. 18, no. 1-2, pp. 18-56, 1990.

[12] A. Cabada and D. R. Vivero, "Expression of the Lebesgue $\Delta$-integral on time scales as a usual Lebesgue integral: application to the calculus of $\Delta$-antiderivatives," Mathematical and Computer Modelling, vol. 43, no. 1-2, pp. 194-207, 2006.

[13] A. Cabada and D. R. Vivero, "Criterions for absolute continuity on time scales," Journal of Difference Equations and Applications, vol. 11, no. 11, pp. 1013-1028, 2005. 
[14] R. P. Agarwal, V. Otero-Espinar, K. Perera, and D. R. Vivero, "Basic properties of Sobolev's spaces on time scales," Advances in Difference Equations, vol. 2006, Article ID 38121, 14 pages, 2006.

[15] C. C. Tisdell, "On first-order discrete boundary value problems," Journal of Difference Equations and Applications, vol. 12, no. 12, pp. 1213-1223, 2006. 